\title{
Dimensionless Film Momentum Transfer Coefficient in Relation to the Analogy among Transport Phenomena
}

\author{
Ryuichi MATSUNO \\ Department of Food Science, Ishikawa Prefectural University, \\ 1-308, Suematsu, Nonoichi-cho, Ishikawa 921-8836, Japan
}

\begin{abstract}
An Mo number, which is a dimensionless formula of the film momentum transfer coefficient $m_{o}$ and reverse of the dimensionless film thickness of flow, was proposed. The Mo numbers deduced from the known theoretical and experimental equations of momentum transfer phenomena for flows in a pipe and around a sphere were compared to the Nusselt numbers, $N u$, and Sherwood numbers, $S h$, for the corresponding heat and mass transfers, respectively, and a good analogy was found among them. The phenomena of momentum transfers were also simply explained using $m_{o}$ and the Mo number. The author expects that these findings might be useful for improving the education of fundamental chemical engineering, food chemical engineering, and biochemical engineering.
\end{abstract}

Key words: Film momentum transfer coefficient, $M o$ number, $N u$ number, Transport phenomena, Analogy

\section{Introduction}

The analogy among transport phenomena has been one of the most interesting subjects in chemical engineering for the author since he learned it as a student.

The fluxes of physical quantities, momentum, energy and mass, are expressed by a unified equation under the condition of constant fluid density.

Flux of physical quantity $=-D_{i f f^{\circ}} \nabla($ Concentration of physical quantity)

where $D_{i f f}$ is a constant having the unit, $\mathrm{cm}^{2} / \mathrm{s}$. The constant $D_{i f f}$ is a dynamic viscosity $\nu$ for momentum transfer, a temperature diffusion coefficient $\alpha$ for heat transfer and a binary diffusion coefficient $D_{A B}$ for mass transfer. With an increase in the $R e$ number, an eddy occurs due to flow instability, bulk flows in a pipe and around an immersed body such as a sphere become turbulent, and concomitantly, boundary film layers of flow (thickness, $\delta$ ) develop along the wall of a pipe and surface of the body in which the fluid has a laminar flow. Due to the same origin, that is flow instability, temperature and concentration boundary film layers with thicknesses $\delta_{t}$ and $\delta_{c}$, respectively, develop. The film heat and mass transfer coefficients are then defined and the heat and mass transfer fluxes at the

(Received 21 Nov. 2005: accepted 16 Jan. 2006)

Fax: +81-76-227-7410, E-mail: matsuno@ishikawa-pu.ac.jp wall or the surface of the immersed body are expressed using the coefficients according to Eq. (1). However, a momentum flux is expressed using the friction coefficient $f$ or drag coefficient $C_{R}$. Why is the momentum flux equation not expressed using a film momentum transfer coefficient $m_{0}$ ? A text book [1] introduced a film momentum transfer coefficient. But it is hardly used for describing the momentum transfer to the best of the author's knowledge.

This short paper introduces a dimensionless film momentum transfer coefficient $M o$ which corresponds to the $N u$ and $S h$ numbers for heat and mass transfers, respectively, and shows how this number behaves in relation to the analogy with heat and mass transfer phenomena [2].

\section{Definition of a Film Momentum Transfer Coefficients $m_{o}$ and its Dimensionless Form Mo Number}

The film momentum transfer coefficient $m_{o}\left[\mathrm{~kg} /\left(\mathrm{m}^{2} \mathrm{~s}\right)\right]$ and $M o$ number [-] are defined by Eqs. (2) and (3), respectively.

$$
\begin{aligned}
& \tau_{w}=-\left.\mu(d u / d r)\right|_{w}=(\mu / \delta)\langle u\rangle=m_{o}\langle u\rangle \\
& M o=m_{o} D / \mu=1 /(\delta / D)
\end{aligned}
$$

where $D$ denotes a representative length such as the diameters of the pipe, sphere, etc., $\mu$, a viscosity, $u$, the fluid 
velocity, $\langle u\rangle$, the average fluid velocity, $r$, the distance in a radial direction and subscript $w$, the wall position.

If we consider the meanings of the $N u$ and $S h$ numbers, Eq. (4) follows.

$$
\begin{aligned}
M o & =1 /(\delta / D)=N u /\left(\delta / \delta_{t}\right)=S h /\left(\delta / \delta_{c}\right) \\
& =M o /(\delta / \delta)
\end{aligned}
$$

For film thicknesses, Eqs. (5) and (6) hold.

$$
\begin{aligned}
& \left(\delta / \delta_{t}\right) \propto f(\operatorname{Pr})=f(\nu / \alpha) \\
& \left(\delta / \delta_{c}\right) \propto f(S c)=f\left(\nu / D_{A B}\right)
\end{aligned}
$$

where $\operatorname{Pr}$ and $S c$ denote the Prandtl and Schmidt numbers, respectively.

\section{Flow in the Pipe}

Equation (2) is applied to the fluid flow in a pipe.

$$
\tau_{w}=-\left.\mu(d u / d r)\right|_{w}=m_{o}\langle u\rangle=\frac{1}{2} \rho\langle u\rangle^{2} f
$$

where $\rho$ is the fluid density.

The $m_{o}$ value is obtained by substituting the well-known correlation between the friction coefficient $f$ and $R e$ number into Eq. (7), and then, the Mo number is deduced from Eq. (3).

For laminar flow:

$$
M o=8 \quad R e<2300
$$

For turbulent flow:

The Blasius formula [3] yields Eq.(9).

$$
M o=0.0395 \operatorname{Re}^{3 / 4} \quad 3 \times 10^{3}<R e<10^{5}
$$

The author first discusses the case of turbulent flow. Equation(9) is the equation to be compared with the correlation of the $N u$ number in turbulent flow [4].

$$
N u=0.023 \operatorname{Re}^{0.8} \operatorname{Pr}^{0.4}
$$

Colburn [2] proposed the $j$ factor and explained the analogy in transport phenomena. If the above result is added, the analogy is denoted by Eq. (11)

$$
j=\frac{1}{2} f \approx \frac{M o}{\operatorname{Re}(\nu / \nu)^{\frac{1}{3}}} \approx \frac{N u}{\operatorname{RePr} r^{\frac{1}{3}}} \approx \frac{S h}{\operatorname{ReSc} c^{\frac{1}{3}}}
$$

Note that the term $(\nu / \nu)^{\frac{1}{3}}$ in the denominator of the third equation is equal to unity.

The $N u$ number for laminar flow is rather complex because it is affected by various factors such as the pipe length, and dependence of the physical properties on the temperature distribution in the pipe. It also depends on the definition of the heat transfer coefficient. The $N u$ number based on a local heat transfer coefficient is comparable to the Mo number for laminar flow (Eq. (8)). The $\mathrm{Nu}$ numbers for following two limiting cases have been also shown to be constant values [5].

For the fully developed laminar flow with the condition of a constant heat flux through the wall :

$$
N u=4.364
$$

For the fully developed laminar flow with the condition of a constant wall temperature :

$$
N u=3.657
$$

The $N u$ numbers, Eqs. (12) and (13), had been calculated by using a flow rate-averaged temperature as the bulk temperature, while Mo number, by using cross sectional area-averaged velocity as the driving force of momentum transfer. The $N u$ number was calculated from the approximate theoretical temperature profile for the former condition proposed by Bird et al. [6] using the cross sectional area-averaged temperature as the bulk temperature and was 6.0 (see Eq. (A.9) in Appendix).

\section{Flow around a Sphere}

The relations between the drag coefficient, $C_{R}$, and $m_{0}$ and the $M o$ number are shown in the following equations.

$$
\begin{aligned}
& m_{o} \pi d_{p}^{2} u=C_{R}\left(\pi d_{p}^{2} / 4\right)\left(\rho u^{2} / 2\right) \\
& M o=m_{o} d_{p} / \mu=\operatorname{Re} C_{R} / 8
\end{aligned}
$$

where $d_{p}$ is the diameter of a sphere, $\rho$, the fluid density and $u$, the approaching velocity.

Using well-known correlations between $C_{R}$ and $R e$,

$$
\begin{array}{lll}
M o=3.0 & R e<6 & \text { Stokes region } \\
M o=1.25 R e^{1 / 2} & 6<R e<10^{5} & \text { Allen region } \\
M o=0.055 R e & 500<R e<10^{5} & \text { Newton region }
\end{array}
$$

These equations are compared to the correlation of $\operatorname{Ran} z$ 
and Marshall [7,8].

$$
N u=2.0+0.6 \operatorname{Re}^{1 / 2} \operatorname{Pr}^{1 / 3}
$$

For $S h$ number, $\operatorname{Pr}$ number is replaced by $S c$ number.

The $M o$ number, $N u$ number and $S h$ number take similar formula and the analogy among the three transfer phenomena holds. However, the numerical coefficients are different. There is a clear reason for this. In the Stokes's law, the drag force arises from two kinds of drag forces, the form and friction drags and their contributions are 1 vs. 2 [9]. The form drag arises from the pressure difference between front and back sides of the sphere against the flow and it does not contribute to heat or mass transfer. Therefore, 2 out of 3 of $M o$ number should be considered for comparison with the $N u$ and $S h$ numbers. In the range of Allen, it is interesting that the contribution of the form drag is increased to $(1 / 2)$ from $(1 / 3)$ for the Stokes range.

\section{Summary}

Although the introduction of $M o$ number is no more than just an idea and is calculated based on the known theoretical and experimental works, it may help students to understand deeply the analogy among the transfer phenomena. Another merit of use of $m_{o}$ and the $M o$ number is that the momentum transport phenomenon is simply explained. "With an increase in $R e$ number, the film thickness decreases, therefore, $m_{0}$ or $M o$ number increases and then, the pressure drop or drag force increases."

\section{Acknowledgement}

The author is grateful to Professor, Dr. Shuichi Yamamoto, Faculty of Engineering, Yamaguchi University for his introduction of the book, " Transport phenomena" [1].

\section{Appendix}

The approximate theoretical temperature profile for the fully developed laminar flow in the pipe with the condition of a constant heat flux through the wall proposed by Bird et al. [6: p.296, Eq.(9.8-31)] is shown as Eq. (A.1).

$$
\Theta=-4 \zeta-\xi^{2}+\frac{1}{4} \xi^{4}+\frac{7}{24}
$$

The dimensionless variables were defined by Eq. (A.2).

$$
\Theta=\frac{T-T_{0}}{q R / k} ; \quad \xi=\frac{r}{R} ; \quad \zeta=\frac{z k}{\rho C_{p} u_{\max } R^{2}}
$$

where $T$ is the temperature, $T_{0}$, the temperature at the inlet of pipe, $z$, the axial direction from the inlet of pipe, $r$, the radial direction from the center of pipe, $R$, the radius of pipe, $q$, the constant heat flux from the pipe wall, $k$, the thermal conductivity, $C_{p}$, the specific heat of fluid and $u_{\max }$ , the maximum fluid velocity.

The denominator of $\Theta$ is rewritten as Eq. (A.3).

$$
q R / k=\frac{h\left(T_{b}-T_{w}\right) R}{k}=\frac{N u}{2}\left(T_{b}-T_{w}\right)
$$

where $h$ is heat transfer coefficient, and the subscripts $b$ and $w$ denote the bulk and wall positions, respectively. Note that $\left(T_{b}-T_{w}\right)$ is the constant driving force of constant heat flux. An another dimensionless temperature $\Theta$ ' is introduced.

$$
\Theta^{\prime}=\frac{T-T_{0}}{T_{b}-T_{w}}=\frac{N u}{2} \Theta
$$

Then, the following relation is hold.

$$
\Theta^{\prime}{ }_{b}-\Theta{ }^{\prime}=1=\frac{N u}{2}\left(\Theta_{b}-\Theta_{w}\right)
$$

Now, if $\Theta_{b}$ and $\Theta_{w}$ are calculated from Eq. (A.1), $N u$ number is calculated from Eq. (A.5).

If the cross sectional area-averaged temperature is chosen as the bulk temperature, $\Theta_{b}$ is calculated as Eq.(A.6).

$$
\begin{aligned}
\Theta_{b}= & \frac{2 \pi \int_{0}^{\mathrm{R}} \Theta r d r}{\pi R^{2}} 2 \int_{0}^{1}\left(-4 \zeta-\xi^{2}+\right. \\
& \left.+\frac{1}{4} \xi^{4}+\frac{7}{24}\right) \xi d \xi=-4 \zeta-\frac{1}{8}
\end{aligned}
$$

$\Theta_{w}$ is calculated straight forwardly.

$$
\Theta_{w}=\Theta(\zeta, 1)=-4 \zeta-\frac{11}{24}
$$

Then,

$$
\Theta_{b}-\Theta_{w}=\frac{1}{3}
$$

By substituting Eq. (A.8) into Eq. (A.5), $N u$ number is deduced as Eq. (A.9).

$$
N u=6.0
$$

In the case of using the flow rate averaged temperature as the bulk temperature, $\left(\Theta_{b}-\Theta_{w}\right)$ becomes as Eq. (A.10). 


$$
\Theta_{b}-\Theta_{w}=\frac{11}{24}
$$

Then, $N u$ number is calculated to be Eq. (12) in the main text.

\section{References}

[1] W. J. Beek, K. M. K. Muttzall; "Transport phenomena”, Chapter 1, A Wiley-Interscience Publication, 1975, p.14.

[2] A. P. Colburn; A method of correlating forced convection heat transfer data and a comparison with fluid friction. Trans. A. I. Ch. E., 29, 174-210 (1933).

[3] H. Schlighting; "Boundary layer theory", Chapter 20,
McGraw-Hill, 1955.

[4] W. H. McAdams; "Heat transmission”, 3rd. Edition, McGraw-Hill Book Co., 1954, p.219.

[5] R. B. Bird, W. E. Stewart, E. N. Lightfoot; "Transport phenomena” , Chapter 13, John Wiley and Sons, Inc., 1960, pp.396-407.

[6] R. B. Bird, W. E. Stewart, E. N. Lightfoot; "Transport phenomena" , Chapter 9, John Wiley and Sons, Inc., 1960, pp.291-297.

[7] W. E. Ranz, W. R. Marshall, Jr.; Evaporation from drops ( I ), Chem. Eng. Prog., 48, 141-146 (1952).

[8] W. E. Ranz, W. R. Marshall, Jr.; Evaporation from drops ( II ), Chem. Eng. Prog., 48, 173-180 (1952).

[9] R. B. Bird, W. E. Stewart, E. N. Lightfoot; "Transport phenomena" , Chapter 2, John Wiley and Sons, Inc., 1960, pp.56-60. 